\title{
Space-time dynamics estimation from space mission tracking data
}

\author{
D. Dirkx ${ }^{1,2}$, R. Noomen ${ }^{1}$, P. N. A. M. Visser ${ }^{1}$, L. I. Gurvits ${ }^{2,1}$, and L. L. A. Vermeersen ${ }^{1}$ \\ 1 Delft University of Technology, Kluyverweg 1, 2629 HS Delft, The Netherlands \\ e-mail: d.dirkx@tudelft.nl \\ 2 Joint Institute for VLBI ERIC, PO Box 2, 7990 AA, Dwingeloo, The Netherlands
}

Received 8 October 2015 / Accepted 21 December 2015

\section{ABSTRACT}

\begin{abstract}
Aims. Many physical parameters that can be estimated from space mission tracking data influence both the translational dynamics and proper time rates of observers. These different proper time rates cause a variability of the time transfer observable beyond that caused by their translational (and rotational) dynamics. With the near-future implementation of transponder laser ranging, these effects will become increasingly important, and will require a re-evaluation of the common data analysis practice of using a priori time ephemerides, which is the goal of this paper.

Methods. We develop a framework for the simultaneous estimation of the initial translational state and the initial proper time of an observer, with the goal of facilitating robust tracking data analysis from next-generation space missions carrying highly accurate clocks and tracking equipment. Using our approach, the influence of physical parameters on both translational and time dynamics are considered at the same level in the analysis, and mutual correlations between the signatures of the two are automatically identified. We perform a covariance analysis using our proposed method with simulated laser data from Earth-based stations to both a Mars and Mercury lander.

Results. Using four years of tracking data for the Mars lander simulations, we find a difference between our results using the simultaneous space-time dynamics estimation and the classical analysis technique (with an a priori time ephemeris) of around $0.1 \%$ in formal errors and correlation coefficients. For a Mercury lander this rises to around 1\% for a one-month mission and $10 \%$ for a four-year mission. By means of Monte Carlo simulations, we find that using an a priori time ephemeris of representative accuracy will result in estimation errors that are orders of magnitude above the formal error when processing highly accurate laser time transfer data.
\end{abstract}

Key words. time - relativistic processes - celestial mechanics - reference systems

\section{Introduction}

By using highly stable space- and Earth-based clocks (e.g. Delva et al. 2012; Bloom et al. 2014), combined with accurate time transfer techniques (e.g. Bjerhammar 1985; Klioner 1992; Petit \& Wolf 1994; Blanchet et al. 2001), the behaviour of distant clocks can be accurately compared. Owing to relativistic clock effects, the data that are obtained from such experiments can be used to improve both geodetic measurements (Müller et al. 2008) and experimental tests of general relativity (Angélil et al. 2014). Important examples of such experiments in ground to space time transfer include the T2L2 project on the Jason-2 satellite (Exertier et al. 2010) and the upcoming ACES project (Delva et al. 2012).

Time transfer data analysis can potentially be complicated by the fact that this analysis is contingent upon accurate knowledge of the state history of the transmitter and the receiver. It was found by Duchayne et al. (2009), however, that the influence of orbital errors on the achievable accuracy of time transfer is acceptably small for the case of the ACES project. Therefore, an a priori orbital solution can be used for the robust analysis of the ACES clock data. Consequently, these data need not (and cannot) be used to improve the orbital solution of the space segment. This approach of decoupled time dynamics and translational dynamics (using an a priori orbital solution for clock data analysis) is typical, and largely sufficient, in the analysis of data from current space missions. However, this is not necessarily true for all current and future time transfer experiments, including those seeking to use relativistic clock effects for improved science return. For instance, it has been found by Delva et al. (2015) that orbital errors of the Galileo 5 and 6 satellites (in eccentric Earth orbits) will manifest themselves in the analysis of the data from the clocks of these satellites. This indicates that the typical approach of using an a priori orbital solution for the clock data analysis is not necessarily the best strategy for all present and future missions.

For cases where the nominal translational dynamics solution is not accurate enough to remove all observable relativistic clock signatures, these clock effects could also be used to improve the orbit determination and parameter estimation quality. However, this requires a suitable inclusion of the clock comparison data in the orbit determination process. Crucially, the clock behaviour needs to be estimated consistently and concurrently with the translational state behaviour. When using the same data types for orbit determination and time transfer, a concurrent analysis of orbital and time behaviour may be especially important. This will be the case for missions employing interplanetary laser ranging (ILR). This technology could be used for range measurements at the $1 \mathrm{~mm}$ to $1 \mathrm{~cm}$ level (Degnan 2002; Turyshev et al. 2010; Dirkx et al. 2014), and time transfer at the resulting 3-30 ps level (assuming sufficiently accurate hardware is used). Very accurate clock models are especially important for missions employing a one-way data type (radio or optical) (Ely et al. 2014; Dirkx et al. 2015), as clock effects (both noise and relativistic) then accumulate over long time periods.

There have been various proposals to exploit space-based clocks to test general relativity, using both near-Earth and 
interplanetary spacecraft (Angélil et al. 2014; Schärer et al. 2014; Deng \& Xie 2013b; Pan \& Xie 2015). These tests could be performed using both optical and radiometric systems. An ILR system, however, will be able to provide the highest accuracy for interplanetary time transfer. The analysis of the performance of these missions requires an analysis of the influence of inaccuracies in the translational dynamics on the analysis of the clock data, as was done for the ACES project by Duchayne et al. (2009). Conversely, it is necessary to analyse the degree to which the clock signal contributes to the estimation of the physical parameters of interest, compared to the measurable influence on the translational dynamics (Deng \& Xie 2013a,b).

Here, we propose a novel general analysis framework in which the estimation of both translational dynamics and proper time dynamics is performed simultaneously, along with the estimation of physical parameters of interest. To achieve this, we extend the typical orbit determination procedure (e.g. Montenbruck \& Gill 2000) to include the estimation of the initial proper times of the observers.

This approach is distinct from the current reduction of space mission tracking data in which the relativistic clock corrections are applied in an a priori manner (or updated iteratively during the orbit determination). Although this approach has been largely sufficient for past and present space missions, future missions (such as those employing ILR) may require a more detailed analysis of the combined influence of relativistic clock effects and translational dynamics on the tracking data.

Our proposed concurrent estimation will allow for a more robust analysis of ranging and time transfer data, preventing the effects of signals from relativistic clock from being incorrectly interpreted as translational dynamics or vice versa. Similarly, it will allow a direct quantification of the correlation between the signal of translational and time dynamics. As has been shown by (e.g. Iorio 2012; Verma et al. 2014; Fienga et al. 2015; Deng \& Xie 2015), a robust quantification of the correlation between the parameters of interest is important in interpreting the estimation results in solar system tests of relativity.

We apply our framework to two interplanetary missions: the tracking of landers on Mars and on Mercury. The goal of our simulations is to demonstrate the application of our proposed methodology. The goal is not to provide a detailed analysis of the scientific performance of such a mission, as has been done by e.g. Hees et al. (2012).

We start by reviewing the relevant details of relativistic celestial mechanics, time dynamics, and the formulation of the time transfer observable in Sect. 2. Our new derivation of the extended variational equations and the partial derivatives of the time transfer observable, including the coupling between translational and time dynamics, is given in Sect. 3. The results of this section comprise the key novel theoretical aspects of this paper. We demonstrate our methodology with numerical simulations of both a Mars and a Mercury lander in Sect. 4, followed by our overall conclusions in Sect. 5.

\section{Dynamics and observable modelling}

In this section we provide an overview of the relevant models that we use in the development of our concurrent estimation in Sect. 3 and our simulations in Sect. 4. We define our notation and conventions in Sect. 2.1. We review the required models for the translational dynamics, time dynamics, and range/time transfer observable in Sects. 2.2-2.4, respectively.

\subsection{Notation and conventions}

We use the $(-+++)$ metric signature and the Einstein summation convention. We use Latin indices $i, j, k=1,2,3$ to denote space indices and Greek indices $\mu, v, \sigma=0,1,2,3$ to denote spacetime indices (with 0 the time index). A subscript ${ }_{, \mu}$ will denote partial differentiation with respect to $x^{\mu}$.

We use the post-Newtonian assumption of slow motion $(v \ll$ $c$ ) and weak fields $\left(\left|g_{\mu \nu}-\eta_{\mu \nu}\right| \ll 1\right)$ since our application of orbit determination is limited to solar system situations. As a result, the metric $g_{\mu \nu}$ can be written in terms of the metric perturbation $h_{\mu v}$ as (Kopeikin et al. 2011)

$g_{\mu v}=\eta_{\mu v}+h_{\mu v}$

with $\eta_{\mu \nu}$ the Minkowski metric. We perform our analysis in the Barycentric Celestial Reference System (BCRS), which is kinematically non-rotating (Kopeikin \& Vlasov 2004).

We use the metric for the solar system at first post-Newtonian order that is recommended by the IAU and discussed by Soffel et al. (2003). However, we note that our overall approach laid out in Sect. 3 is not limited to this particular choice of metric, or even to metric theories of gravity.

\subsection{Relativistic celestial mechanics}

In this section we review the aspects of relativistic celestial mechanics necessary for our subsequent discussion. Further extensive discussion on the topic can be found in e.g. Brumberg (1972), Soffel (1989), Brumberg (1991), Kopeikin \& Vlasov (2004), Kopeikin et al. (2011).

The general four-dimensional equation of motion of a point mass experiencing only gravitational forces is (Kopeikin et al. 2011)

$\frac{\mathrm{d}^{2} x^{\mu}}{\mathrm{d} \tau^{2}}=-\Gamma_{\alpha \beta}^{\mu} \frac{\mathrm{d} x^{\alpha}}{\mathrm{d} \tau} \frac{\mathrm{d} x^{\beta}}{\mathrm{d} \tau}$

$\Gamma_{\alpha \beta}^{\mu}=\frac{1}{2} g^{\mu v}\left(g_{v \alpha, \beta}+g_{v \beta, \alpha}-g_{\alpha \beta, v}\right)$,

where $x^{\mu}$ denotes the four-position $\{c t, x, y, z\}, \tau$ denotes the proper time of an observer co-moving with the test body, and $\Gamma_{\alpha \beta}^{\mu}$ denotes the Christoffel symbols of the metric connection. We apply this equation in this article and note that the additional terms that arise owing to non-gravitational forces or self-gravitation (Kopeikin \& Vlasov 2004) can be included when a more detailed dynamical model is required.

We also note that in certain alternative theories of gravity, the formulation of Eqs. (2) and (3) are different (Kopeikin et al. 2011), and additional terms need to be included. The inclusion of these terms results in a modification of the equations that we use and derive further in this article. However, it does not change the general methodology that is derived in Sect. 3.

The equations of motion given by Eq. (2) can be written in terms of a coordinate time $t$ (such as Barycentric Coordinate Time TCB) instead of proper time $\tau$. This yields equations of motion expressed in a timescale typically used in orbit propagation and determination, for example (Kopeikin et al. 2011)

$$
\begin{aligned}
\ddot{x}^{i}=-\Gamma_{\alpha \beta}^{i} \dot{x}^{\alpha} \dot{x}^{\beta}+\frac{1}{c} \Gamma_{\alpha \beta}^{0} \dot{x}^{\alpha} \dot{x}^{\beta} \dot{x}^{i} \\
=-c^{2} \Gamma_{00}^{i}-2 c \Gamma_{0 j}^{i} \dot{x}_{j}-\Gamma_{j k}^{i} \dot{x}^{j} \dot{x}^{k}+\dot{x}^{i} \cdot \ldots \\
\ldots \cdot\left(c \Gamma_{00}^{0}+2 \Gamma_{0 j}^{0} \dot{x}^{j}+\frac{1}{c} \Gamma_{j k}^{0} \dot{x}^{j} \dot{x}^{k}\right),
\end{aligned}
$$


where the overdot denotes a derivative with respect to coordinate time $t$.

In numerical ephemerides of solar system bodies, the independent variable is typically Dynamical Barycentric Time (TDB), which is a scaled version of TCB and not a coordinate time itself. In the remainder of this paper, we assume that the independent variable $t$ is the coordinate time TCB. A detailed discussion on the consequences of using TDB instead of TCB is given by Klioner (2008).

\subsection{Proper time dynamics}

Eq. (2) describes the evolution of the four-velocity $\mathrm{d} x^{\mu} / \mathrm{d} \tau$ of an observer; the first equation $(\mu=0)$ describes the evolution of the time component and the other three equations $(\mu=1,2,3)$ describe the translational dynamics. The norm of the four-velocity is constant by definition, (e.g. Misner et al. 1973), a condition that can be expressed as follows using Eq. (1):

$\frac{\mathrm{d} \tau}{\mathrm{d} t}=\sqrt{-g_{\mu \nu} \frac{\dot{x}^{\mu} \dot{x}^{\nu}}{c^{2}}}=\sqrt{1-\frac{v^{2}}{c^{2}}-h_{\mu \nu} \frac{\dot{x}^{\mu} \dot{x}^{\nu}}{c^{2}}}=1+O\left(c^{-2}\right)$.

Since the leading-order difference between proper time rate and coordinate time rate is at the $c^{-2}$ level, we propagate the proper time difference $\Delta \tau$ of an observer:

$\Delta \tau(t)=\tau(t)-t$.

To evaluate the derivative of Eq. (7) with respect to $t$ using Eq. (6), we expand it using a Taylor-series, thereby canceling the terms of $O(1)$. This prevents rounding errors from limiting the precision of our results. The order of this expansion is chosen such that the error that it introduces is below numerical precision. For a first-order expansion:

$\frac{\mathrm{d} \Delta \tau}{\mathrm{d} t}=-\frac{1}{2}\left(\frac{v^{2}}{c^{2}}+h_{\mu v} \frac{\dot{x}^{\mu} \dot{x}^{v}}{c^{2}}\right)$.

By numerically integrating Eqs. (5) and (8), we obtain a numerical solution for the space-time behaviour of a given observer. This is equivalent to the approach taken in the generation of ephemerides by Fienga et al. (2009).

\subsection{Time transfer observable}

To include time dynamics in the estimation, measurements of the relative proper time rates of separated clocks are required. These measurements can be realized by time transfer. In this section, we summarize the models we use to simulate time transfer observables, with extensive details provided by e.g. Klioner (1992), Petit \& Wolf (1994) and Blanchet et al. (2001).

We consider two clocks between which time transfer is performed by means of electromagnetic signal transmission, for instance laser pulses. The signal is transmitted by the transmitter (station $A$ ) at the proper time $\tau^{(A)}\left(t_{1}\right)$ and coordinate time $t_{1}$ and received by the receiver (station $B$ ) at its proper time $\tau^{(B)}\left(t_{2}\right)$ and coordinate time $t_{2}$. In the following, we denote $\tau^{(x)}\left(t_{i}\right)$ as $\tau_{i}^{(x)}$. The one-way time transfer observable $h_{\tau}^{(1)}$ is then the difference between the two proper times, (e.g. Duchayne et al. 2009):

$h_{\tau}^{(1)}=\tau_{2}^{(B)}-\tau_{1}^{(A)}$.

The proper time rate, given by Eq. (6), will be different for the observers $A$ and $B$. This causes further variability of the observable $h_{\tau}^{(1)}$ beyond that, which is caused by the translational (and rotational) dynamics of the receiving and transmitting bodies.
The time transfer observable is calculated by using the numerically integrated translational dynamics and proper times of both the receiver and transmitter from Eqs. (8) and (5):

$$
\begin{aligned}
& h_{\tau}^{(1)}\left(\tau_{1}^{(A)}\right)=\tau_{2}^{(B)}-\tau_{1}^{(A)} \\
& t_{1}=t_{1}\left(\tau_{1}^{(A)}\right) \\
& t_{2}=t_{1}+T_{21}\left(t_{1} ; \boldsymbol{x}_{t}(t), \boldsymbol{x}_{r}(t)\right) \\
& \tau_{2}^{(B)}=\tau_{2}^{(B)}\left(t_{2}\right) .
\end{aligned}
$$

In the above $T_{21}$ denotes the classical one-way light-time observable (Moyer 2005),

$T_{21}\left(t_{1}\right)=\frac{\left|\boldsymbol{x}_{B}\left(t_{1}+T_{21}\right)-\boldsymbol{x}_{A}\left(t_{1}\right)\right|}{c}+\Delta T_{21}\left(t_{1}, t_{2}, \boldsymbol{x}_{1}, \boldsymbol{x}_{2}\right)$,

where the term $\Delta T_{21}$ denotes light time corrections due to atmospheric and relativistic effects. The model for the one-way range observable that is typically used in orbit determination is calculated from $c T_{21}$, and clearly differs from the one-way time transfer observable $h_{\tau}^{(1)}$ by virtue of Eqs. (11) and (13). We note that the time transfer observable is referenced to a proper time at one of the link ends, whereas the range observable is referenced to one of the coordinate times (Hees et al. 2012).

The observable $h_{\tau}^{(1)}$ can be related to the one-way light time as

$h_{\tau}^{(1)}=T_{21}\left(t_{1}\left(\tau_{1}^{(A)}\right)\right)-\int_{t_{0}}^{t_{1}} \frac{\mathrm{d} \Delta \tau^{(A)}}{\mathrm{d} t} \mathrm{~d} t+\int_{t_{0}}^{t_{2}} \frac{\mathrm{d} \Delta \tau^{(B)}}{\mathrm{d} t} \mathrm{~d} t$,

where $t_{0}$ denotes the reference time at which the initial conditions for the numerical solution of Eqs. (5) and (8) are specified. From this equation, it is clear that the integrated value of $\Delta \tau$ over long time periods at both the transmitter and receiver influences the observable.

When using both an up- and downlink one-way range measurement to construct a two-way observable $h_{\tau}^{(2)}$, for instance using an asynchronous active laser ranging system (Degnan 2002; Birnbaum et al. 2010), the influence of relativistic clock effects is substantially reduced in the same manner that the influence of clock noise at both link ends is substantially reduced (Dirkx et al. 2015). The downlink of a two-way measurement from the space segment to the ground station consists of a signal transmitted by the space segment at coordinate time $t_{3}$, where $t_{3}=t_{2}+\delta t$, with $\delta t$ typically at most of the order of seconds. This signal is then received by the ground station at coordinate time $t_{4}$. The raw two-way light time, as measured by the ground station, then becomes

$$
\begin{aligned}
h_{\tau}^{(2)} & =\tau_{4}^{(A)}-\tau_{1}^{(A)} \\
& =T_{21}\left(t_{1}\left(\tau_{1}^{(A)}\right)\right)+T_{43}\left(t_{3}\right)+\int_{t_{1}}^{t_{4}} \frac{\mathrm{d} \Delta \tau^{(A)}}{\mathrm{d} t} \mathrm{~d} t+\delta t \\
t_{3} & =t_{1}\left(\tau_{1}^{(A)}\right)+T_{21}\left(t_{1}\left(\tau_{1}^{(A)}\right)\right)+\delta t .
\end{aligned}
$$

Since the retransmission time at the space segment $\delta t$ is small, the influence of the proper time rate of the space segment on $h_{\tau}^{(2)}$ is almost completely removed. However, the conversion from $h_{\tau}^{(2)}$ to the two-way coordinate light time $t_{4}-t_{1}$ remains sensitive to the difference in rates between $t$ and $\tau^{(A)}$, but only as integrated over $t_{4}-t_{1}$. Comparing Eqs. (15) and (17), it is clear that the oneway observable is sensitive to clock effects accumulated over long periods of time, whereas the two-way observable is largely insensitive to relativistic clock variations. Nevertheless, since an 
asynchronous two-way observable simply consists of two oneway observables, it can be used to exploit relativistic clock effects during the estimation by including one or both of the oneway time transfer observables separately in the estimation.

Since our purpose in this work is to present a model for estimation of the space-time dynamics, we do not provide detailed models for relativistic effects on the $\Delta T_{21}$ component of time transfer. Such models have been developed for a variety of contexts and applications (e.g. Klioner 1992; Kopeikin \& Schäfer 1999; Linet \& Teyssandier 2002), but their inclusion does not alter the formulation of our estimation framework. The inclusion of these effects slightly changes the exact light-time and therefore causes a small modification of $t_{2}$ and $\tau_{2}^{B}$ for a one-way range observable, see Eq. (12). However, neither the translational nor the time dynamics of the observers is influenced by these effects.

\section{Coupled space-time estimation}

In this section, we present our proposed approach for the estimation of coupled space-time dynamics. We reiterate that this approach may become important for near-future situations where the same data are used for the estimation of the translational dynamics and the characterization of relativistic clock effects. We start by briefly outlining the problem in Sect. 3.1. We then derive variational equations for the coupled estimation in Sect. 3.2 and the complete partial derivatives of the time transfer observables in Sect. 3.3.

\subsection{Proper time estimation}

To estimate the orbit of a celestial body or artificial satellite from tracking data, the initial state of that body is adjusted in such a manner that the weighted r.m.s. (root mean square) difference between the measured and modelled observables is minimized (e.g. Montenbruck \& Gill 2000). Additionally, parameters that influence the force and/or observation models can be concurrently estimated.

By extending this approach to the coupled space-time dynamics case, we include the estimation of the initial proper time difference(s) $\Delta \tau$ of one or more observers. The value of $\Delta \tau\left(t_{0}\right)=\Delta \tau_{0}$ of these observers can then be estimated by minimizing the residuals between the modelled and measured values of $\Delta \tau(t)$, as well as any other parameters that influence the solution of Eq. (7), in the same manner as is done in translational orbit determination (e.g. Montenbruck \& Gill 2000).

This approach has an advantage: it removes the issue that relativistic clock signals could be interpreted as translational dynamics without this effect being quantified by the estimator. Furthermore, it provides a manner in which the capabilities of relativistic clock signals can be robustly analysed in order to enhance the science return from space mission tracking data (Mayrhofer \& Pail 2012; Angélil et al. 2014; Schärer et al. 2014). Crucially, the adjustment of the proper time estimation is done concurrently with that of the translational state behaviour, allowing correlations between the two to be identified and quantified.

\subsection{Coupled variational equations}

We derive a set of equations to estimate the initial state of $N$ bodies and the proper time differences of $M$ observers. We denote the translational state of a single body $n$ as $\boldsymbol{y}_{n}=\left(\boldsymbol{x}_{n} ; \dot{\boldsymbol{x}}_{n}\right)$, with $\boldsymbol{x}_{n}$ the current position of body $n$ and $\dot{\boldsymbol{x}}_{n}$ its velocity.
The concatenated vector of all states is denoted as $\boldsymbol{Y}$; so that $\boldsymbol{Y}=\left(\boldsymbol{y}_{1} ; \ldots ; \boldsymbol{y}_{N}\right)$. The concatenated vector of all proper time differences is denoted as $\boldsymbol{T}$, so that $\boldsymbol{T}=\left(\Delta \tau_{1} ; \ldots ; \Delta \tau_{M}\right)$. Finally, we construct our full state vector $\boldsymbol{X}$ as follows:

$\boldsymbol{X}=\left(\begin{array}{c}\boldsymbol{Y} \\ \boldsymbol{T}\end{array}\right)=\left(\boldsymbol{y}_{1} \ldots \boldsymbol{y}_{N}, \Delta \tau_{1} \ldots \Delta \tau_{M}\right)^{T}$.

Our goal in this section is to derive equations to determine the linearized change in $\boldsymbol{X}$ due to a change in its initial value $\boldsymbol{X}\left(t_{0}\right)$ (denoted $\boldsymbol{X}_{0}$ ) and the model parameter vector $\boldsymbol{p}$. This is in contrast to the typical approach to orbit determination and time transfer where the adjustment of $\boldsymbol{Y}_{0}$ to the data is performed using an a priori fixed $\boldsymbol{T}_{0}$.

These changes are encoded in the state transition matrix $\Phi\left(t ; t_{0}\right)$ and sensitivity matrix $S(t)$ (Montenbruck \& Gill 2000). Extending these matrices to our case, using Eq. (19) as a state vector, we obtain the following:

$$
\begin{aligned}
& \Phi\left(t ; t_{0}\right)=\frac{\partial \boldsymbol{X}(t)}{\partial \boldsymbol{X}_{0}}=\left(\begin{array}{l}
\frac{\partial \boldsymbol{Y}(t)}{\partial \boldsymbol{Y}_{0}} \frac{\partial \boldsymbol{Y}(t)}{\partial \boldsymbol{T}_{0}} \\
\frac{\partial \boldsymbol{T}(t)}{\partial \boldsymbol{Y}_{0}} \frac{\partial \boldsymbol{T}_{(t)}}{\partial \boldsymbol{T}_{0}}
\end{array}\right) \\
& S(t)=\frac{\partial \boldsymbol{X}(t)}{\partial \boldsymbol{p}}=\left(\begin{array}{c}
\frac{\partial \boldsymbol{Y}(t)}{\partial \boldsymbol{p}} \\
\frac{\partial \boldsymbol{T}(t)}{\partial \boldsymbol{p}}
\end{array}\right) .
\end{aligned}
$$

These partial derivatives denote the change in the state $\boldsymbol{X}$ that would occur if the initial state or model parameter were changed.

The differential equation describing the behaviour of $\Phi\left(t, t_{0}\right)$ and $S(t)$ is given by (Montenbruck \& Gill 2000). Here, we generalize their result to the estimation of $N$ translational states and $M$ observer proper times. This results in the following formulation for the variational equations

$$
\begin{aligned}
\frac{\mathrm{d}}{\mathrm{d} t}\left(\Phi\left(t ; t_{0}\right), S(t)\right)= & \left(\begin{array}{l}
\frac{\partial \dot{\boldsymbol{Y}}(t)}{\partial \boldsymbol{Y}} \frac{\partial \dot{\boldsymbol{Y}}(t)}{\partial \boldsymbol{T}} \\
\frac{\partial \boldsymbol{T}(t)}{\partial \boldsymbol{Y}} \frac{\partial \boldsymbol{T}(t)}{\partial \boldsymbol{T}}
\end{array}\right)\left(\Phi\left(t, t_{0}\right), S(t)\right)+\ldots \\
& \ldots+\left(\begin{array}{l}
0_{6 N \times(6 N+M)} \frac{\partial \dot{\boldsymbol{Y}}(t)}{\partial \boldsymbol{p}} \\
0_{M \times(6 N+M)} \frac{\partial \boldsymbol{T}(t)}{\partial \boldsymbol{p}}
\end{array}\right) \\
& =A(\boldsymbol{X}, \dot{\boldsymbol{X}}, t)\left(\Phi\left(t, t_{0}\right), S(t)\right)+B(\boldsymbol{X}, \dot{\boldsymbol{X}}, t)
\end{aligned}
$$

$\Phi\left(t_{0}, t_{0}\right)=1_{(6 N+M) \times(6 N+M)}$

$S\left(t_{0}\right)=0_{(6 N+M) \times N_{\mathrm{P}}}$

where $N_{\mathrm{P}}$ denotes the length of the estimated parameter vector $\boldsymbol{p}$.

There is no explicit dependency of $\dot{\boldsymbol{Y}}$ on any of the observers' proper times $\boldsymbol{T}$, only on the coordinate time $t$. Similarly, the metric in the global coordinates does not depend explicitly on the proper time of a specific observer (Soffel et al. 2003). As a result, there is also no explicit dependency of $\dot{\boldsymbol{T}}$ on $\boldsymbol{T}$ and the matrix $A(\boldsymbol{X}, \dot{\boldsymbol{X}}, t)$ reduces to

$A(\boldsymbol{X}, \dot{\boldsymbol{X}}, t)=\left(\begin{array}{cc}\frac{\partial \dot{\boldsymbol{Y}}(t)}{\partial \boldsymbol{Y}} & 0 \\ \frac{\partial \dot{\boldsymbol{T}}(t)}{\partial \boldsymbol{Y}} & 0\end{array}\right)$.

An adjustment of $\boldsymbol{T}_{0}$ will only result in a change in $\boldsymbol{T}(t)$, not in $\boldsymbol{Y}(t)$. However, an adjustment of $\boldsymbol{Y}_{0}$ of $\boldsymbol{p}$ influences both $\boldsymbol{Y}(t)$ and $\boldsymbol{T}(t)$, introducing the space-time dynamics coupling into the estimation.

The expression for the top left matrix block of $A(\boldsymbol{X}, \dot{\boldsymbol{X}}, t)$ is composed of the blocks containing the derivative of the state of body $m$ with respect to the state of body $n$ as follows:

$\frac{\partial \dot{\boldsymbol{Y}}_{m}(t)}{\partial \boldsymbol{Y}_{n}(t)}=\left(\begin{array}{cc}0_{3 \times 3} & \delta_{m n} 1_{3 \times 3} \\ \frac{\partial \dot{\boldsymbol{x}}_{m}}{\partial \boldsymbol{x}_{n}} & \frac{\partial \ddot{\boldsymbol{x}}_{m}}{\partial \dot{\boldsymbol{x}}_{n}}\end{array}\right)$ 
To evaluate the components of the variational equations, the partial derivatives of the accelerations and proper time rates with respect to the full parameter vector $\boldsymbol{q}\left(=\left(\boldsymbol{X}_{0} ; \boldsymbol{p}\right)\right)$ must be evaluated. For the equations of motion in the form of Eq. (4), this results in the general formulation

$$
\begin{aligned}
\frac{\partial \ddot{x}^{i}}{\partial \boldsymbol{q}} & =-\frac{\partial \Gamma_{\alpha \beta}^{i}}{\partial \boldsymbol{q}} \dot{x}^{\alpha} \dot{x}^{\beta}+\frac{1}{c} \frac{\partial \Gamma_{\alpha \beta}^{0}}{\partial \boldsymbol{q}} \dot{x}^{\alpha} \dot{x}^{\beta} \dot{x}^{i}-2 \Gamma_{\alpha \beta}^{i} \frac{\partial \dot{x}^{\alpha}}{\partial \boldsymbol{q}} \dot{x}^{\beta}+\ldots \\
& \ldots+\frac{1}{c} \Gamma_{\alpha \beta}^{0}\left(\dot{x}^{\alpha} \dot{x}^{\beta} \frac{\partial \dot{x}^{i}}{\partial \boldsymbol{q}}+2 \frac{\partial \dot{x}^{\alpha}}{\partial \boldsymbol{q}} \dot{x}^{\beta} \dot{x}^{i}\right),
\end{aligned}
$$

where the symmetry of the Christoffel symbols in the indices $\alpha \beta$ has been exploited.

Estimation of the initial proper time difference requires the calculation of the partial derivative of the proper time rate $\mathrm{d} \tau / \mathrm{d} t$ with respect to $\boldsymbol{q}$. These partial derivative are derived from Eq. (6):

$$
\frac{\partial \dot{\tau}}{\partial \boldsymbol{q}}=\frac{1}{2 \dot{\tau}}\left(\frac{\partial g_{\mu v}}{\partial \boldsymbol{q}} \dot{x}^{\mu} \dot{x}^{v}+2 g_{\mu \nu} \dot{x}^{\mu} \frac{\partial \dot{x}^{\mu}}{\partial \boldsymbol{q}}\right)
$$

The partial derivatives of the Christoffel symbols, required for the evaluation of Eq. (28) can be obtained numerically or analytically from Eq. (3) as follows:

$$
\begin{aligned}
\frac{\partial \Gamma_{\alpha \beta}^{\mu}}{\partial \boldsymbol{q}}= & \frac{1}{2}\left(-\frac{\partial h^{\mu v}}{\partial \boldsymbol{q}}\left(h_{v \alpha, \beta}+h_{\nu \beta, \alpha}-h_{\alpha \beta, v}\right)+\ldots\right. \\
& \left.\ldots+g^{\mu \nu}\left(\frac{\partial h_{\nu \alpha, \beta}}{\partial \boldsymbol{q}}+\frac{\partial h_{\nu \beta, \alpha}}{\partial \boldsymbol{q}}-\frac{\partial h_{\alpha \beta, \nu}}{\partial \boldsymbol{q}}\right)\right) .
\end{aligned}
$$

From these equations, the variational equations can be evaluated analytically or numerically from only the first and second derivatives of the metric perturbation, in addition to the current state $\boldsymbol{Y}(t)$ and the metric itself.

\subsection{Time transfer observable partials}

To incorporate the time transfer observable into the orbit determination, we require the partial derivatives of the $h_{\tau}^{(1)}$ observable, given by Eq. (9), with respect to the full state vector $\boldsymbol{X}$ and the model parameters $\boldsymbol{p}$. Denoting an arbitrary combination of entries of these vectors by $\boldsymbol{q}$, the following is needed:

$\frac{\partial h_{\tau}^{(1)}}{\partial \boldsymbol{q}}=\frac{\partial \tau_{2}^{(B)}}{\partial \boldsymbol{q}}-\frac{\partial \tau_{1}^{(A)}}{\partial \boldsymbol{q}}$

As discussed in Sect. 2.4, we reference the time transfer to either the transmission or reception proper time. Here, we again show the case where we reference the observation to the transmission time, so that $h_{\tau}^{(1)}=h_{\tau}^{(1)}\left(\tau_{1}^{(A)}\right)$, with a fixed $\tau_{1}^{(A)}$. This makes the second term on the right-hand side of Eq. (31) equal to zero. The first term on the right-hand side then becomes

$$
\begin{aligned}
\frac{\partial \tau_{2}^{(B)}}{\partial \boldsymbol{q}} & =\dot{\tau}_{2}^{(B)} \frac{\partial t_{2}}{\partial \boldsymbol{q}}+\left.\frac{\partial \tau_{2}^{(B)}}{\partial \boldsymbol{q}}\right|_{t_{2}=\text { const. }} \\
& =\dot{\tau}_{2}^{(B)} \frac{\partial t_{2}}{\partial \boldsymbol{q}}+\frac{\partial \Delta \tau_{2}^{(B)}}{\partial \boldsymbol{q}}
\end{aligned}
$$

The first term on the right-hand side of these equations represents the change in reception proper time due to a change in reception coordinate time, and the second term represents the inherent change in the receiver proper time, i.e. the direct influence on the integrated result of Eq. (8). The reception coordinate time partial is expanded as follows from Eq. (12):

$\frac{\partial t_{2}}{\partial \boldsymbol{q}}=\frac{\partial t_{1}}{\partial \boldsymbol{q}}+\frac{\partial T_{21}}{\partial \boldsymbol{q}}+\frac{\partial T_{21}}{\partial t} \frac{\partial t_{1}}{\partial \boldsymbol{q}}$

The partial derivative of the transmission coordinate time $t_{i}$ is obtained from Eq. (7),

$$
\frac{\partial t_{1}}{\partial \boldsymbol{q}}=-\frac{\partial \Delta \tau_{1}^{(A)}}{\partial \boldsymbol{q}}
$$

since $\tau_{1}^{(A)}$ is kept fixed. Combining these results yields

$$
\left.\frac{\partial h_{\tau}^{(1)}}{\partial \boldsymbol{q}}\right|_{\tau_{1}^{(A)}=\text { const. }}=\frac{\partial \Delta \tau_{2}^{(B)}}{\partial \boldsymbol{q}}-\frac{\partial \Delta \tau_{1}^{(A)}}{\partial \boldsymbol{q}}\left(1+\dot{T}_{21}\right) \dot{\tau}_{2}^{(B)}+\frac{\partial T_{21}}{\partial \boldsymbol{q}} \dot{\tau}_{2}^{(B)}
$$

The final term of this equation, $\frac{\partial T_{21}}{\partial q}$, is the one-way range partial, as expanded explicitly by e.g. Moyer (2005). The first two terms on the right-hand side are the scaled terms of the extended state transition matrix, as obtained by numerical integration of Eq. (22). It is these terms that our derivation has shown need to be added to the estimation to exploit the coupling between the space and time dynamics.

As a result of the formulation of Eq. (36) and the fact that $\dot{X}$ is independent of $\boldsymbol{T}$, the partial derivatives of the observations with respect to the initial proper times behave largely like those of range biases. Deviations from unity of the partial derivatives $\frac{\partial h}{\partial T}$ instead follow from the $\left(1+\dot{T}_{21}\right) \dot{\tau}_{r}$ term in Eq. (36).

Equation (36) can be used to determine the direct part of the sensitivity of the observable to a change in $\boldsymbol{p}$ and $\boldsymbol{Y}$. To compute the total sensitivity to $\boldsymbol{Y}_{0}$ and $\boldsymbol{p}$, the state transition and sensitivity matrices that are obtained by numerical integration of Eq. (22) are used to obtain the following:

$$
\begin{aligned}
& \frac{\partial h(t)}{\partial \boldsymbol{X}_{0}}=\frac{\partial h}{\partial \boldsymbol{X}} \Phi\left(t, t_{0}\right) \\
& \frac{\partial h(t)}{\partial \boldsymbol{p}}=\left.\frac{\partial h}{\partial \boldsymbol{p}}\right|_{X=\text { const. }}+\frac{\partial h}{\partial \boldsymbol{X}} S(t) .
\end{aligned}
$$

These partial derivatives are calculated for each of the observations, from which the least-squares parameter adjustment can be performed, as discussed in greater detail by e.g. Montenbruck \& Gill (2000).

\section{Test cases: Mars and Mercury landers}

We illustrate the methodology we describe in Sect. 3 with numerical simulations of the Mars and Mercury lander missions performing laser ranging and time transfer to Earth.

The simulations we present are not a detailed analysis and assessment of the performance of the missions that are considered, as is performed by e.g. Turyshev et al. (2010) and Hees et al. (2012). They are included here to show the potential influence of the relativistic clock effects on the estimation procedure for the case of representative planetary lander missions using laser ranging. We analyse both the Mars and Mercury lander missions to assess the influence of the depth of the space segment inside the gravity well of the Sun. Simulations of tracking data to planetary landers have been performed for a wide variety of missions and tracking data types, e.g. (Turyshev et al. 2010; Dehant et al. 2011; Dirkx et al. 2014). Also, models and analyses 
of time transfer at interplanetary distances have been performed by e.g. (Degnan 2002; Nelson 2011; Pan \& Xie 2015). However, none of these simulations have included the coupling between translational dynamics and relativistic clock effects. It is the goal of the present paper to present the influence of this coupling. For an Earth-orbiting mission, however, a sensitivity analysis of this coupling was performed by (Duchayne et al. 2009) using an approach different from the one derived here. Their analysis provides a rigorous sensitivity analysis of the time transfer data for the ACES mission. However, the methods they used do not provide a framework in which the contribution of the clock data to the orbit determination and parameter estimation can be quantified.

In Sect. 4.1 we discuss the settings of our simulations. Subsequently, we present a covariance analysis of the performance of the lander missions in Sect. 4.2. There, we consider both the cases with and without the space-time dynamics coupling. Finally, we compare the influence of proper time estimation with the influence of a priori and iterative a posteriori clock (re)calibration in Sect. 4.3.

\subsection{Simulation settings}

The settings for our simulations are the following. During one year of operations, we simulate one 30-min arc of one-way laser ranging data per day from each of the four Earth-based International Laser Ranging Service (ILRS) stations (Pearlman et al. 2002), where we simulate a single observation per minute. From these simulated data, we estimate the following parameters using a least-squares approach (e.g. Montenbruck \& Gill 2000):

- PPN parameters $\beta$ and $\gamma$. Nominal values equal to 1 in general relativity.

- Solar $J_{2}$ gravity field coefficient $J_{2} \odot$ (quadrupole moment). Nominal value $\approx 2.2 \times 10^{-7}$ (Mecheri et al. 2004).

- Mars/Mercury initial translational state $\boldsymbol{y}_{M, 0}$, nominal values taken from DE430 ephemeris (Folkner et al. 2014).

- Initial proper time difference between space segment and each Earth station $i$, denoted $\Delta \tau^{S C}\left(t_{0}\right)-\Delta \tau^{(E, i)}\left(t_{0}\right)$.

- Mars/Mercury-fixed lander position $r_{L}^{(M)}$ (both placed equatorially).

To decorrelate the PPN parameter $\gamma$ from the other parameters, its influence on the light-time correction $\Delta T_{21}$ is crucial. Here, we include the Sun's first-order effect on light time, as given by e.g. Moyer (2005). Light time-corrections due to other effects are sufficiently small to be neglected in our conceptual analysis (Minazzoli \& Chauvineau 2011).

We do not estimate empirical clock noise parameters, as is done by Dirkx et al. (2015), as we assume that the systems are equipped with highly accurate clocks, for which the stochastic clock noise is minimal. Nevertheless, it should be understood that the detection of long-period signals requires highly stable clocks. To be able to observe a clock effect that manifests itself as a $1 \mathrm{~cm}$ change in $c \cdot h_{\tau}^{(1)}$ over a period of 1 day requires an Allan deviation of about $4 \times 10^{-16}$ over such an integration time, which is about a factor of 2 better than that of the proposed deep space atomic clock (DSAC; Prestage \& Weaver 2007). For a similar $1 \mathrm{~cm}$ change over a period of 1 year, an Allan deviation of the clock of $10^{-18}$ over a similar period is required, which may be facilitated in the future by optical clocks (e.g. Bloom et al. 2014). A detailed analysis of the error sources of interplanetary laser ranging is given by Dirkx (2015).
Summarizing, our simulations are performed as follows:

- The translational and time dynamics for each observer are concurrently numerically integrated using Eqs. (4) and (8), with the full metric given by Soffel et al. (2003). We include the point-mass potential of the Earth and Mars/Mercury and the Sun's gravity field including its $J_{2}$ term.

- From the simulated space-time dynamics, we use Eq. (9) to simulate the observables between the space segment and the ground stations. The observations are constrained by a $15^{\circ}$ minimum elevation angle and a $5^{\circ}$ Sun avoidance angle.

- We numerically integrate the state transition and sensitivity matrices using Eq. (22). We obtain the partial derivatives of the Christoffel symbols, which are required for evaluating Eq. (30), by means of numerical differentiation.

- Using Eq. (36), we calculate the direct contribution of the partial derivatives of the observations with respect to the estimated parameters. Subsequently, we use the numerically integrated $\Phi\left(t, t_{0}\right)$ and $S(t)$ matrices to calculate the complete partial derivative of each observation with respect to each of the estimated parameters using Eqs. (37) and (38).

- From the partial derivatives of the observations, we set up the information matrix $H$, from which we calculate the covariance matrix (Montenbruck \& Gill 2000).

For the Mercury lander, we evaluate two different mission lengths. Since Mercury's rotation is in a 3:2 resonance with its orbit, a Mercury lander experiences long periods of complete darkness and intense sunlight. Therefore, a four-year Mercury lander mission is highly challenging with current technological and budgetary constraints. A 30-day mission, however, would be easier to realize as it is possible to keep the lander under the same illumination conditions for this amount of time while retaining constant visibility to the Earth. Nevertheless, the depth of Mercury in the Sun's gravity well makes it an attractive test case for exploiting relativistic effects. Since our primary goal in this section is to get a broad overview of the potential effect of space-time dynamics coupling on range data inversion, we will analyse both a 30-day and a 4-year mission, acknowledging the technological difficulties associated with a four-year mission.

\subsection{Covariance analysis results}

We perform a covariance analysis to investigate the effect of the addition of the $\Delta \tau$ terms in Eq. (36), as well as the addition of the estimation of $\Delta \tau^{S C}\left(t_{0}\right)-\Delta \tau^{E, i}\left(t_{0}\right)$ on the formal error and correlation statistics (Montenbruck \& Gill 2000). We present the results of the covariance analyses of the Mars and Mercury lander estimations with the settings defined in Sect. 4.1. By using a covariance analysis, we implicitly assume that our truth and estimation models are equal, and that our observations have errors that are identically and independently distributed with a zeromean Gaussian distribution. A more detailed analysis of the error sources in planetary laser ranging, and methods in which to mitigate them, is given by Dirkx (2015).

The formal error obtained from the covariance analysis is more optimistic than the true error that is obtained from real data (e.g. Marty et al. 2009; Konopliv et al. 2011; Dirkx et al. 2014). However, by comparing the formal error with and without the contribution of the space-time coupling terms, we identify the relative sensitivity of the solution to the inclusion of the coupled space-time dynamics estimation that we propose here. Furthermore, our covariance analysis will produce the change in the correlations between the estimation of the parameters due to 


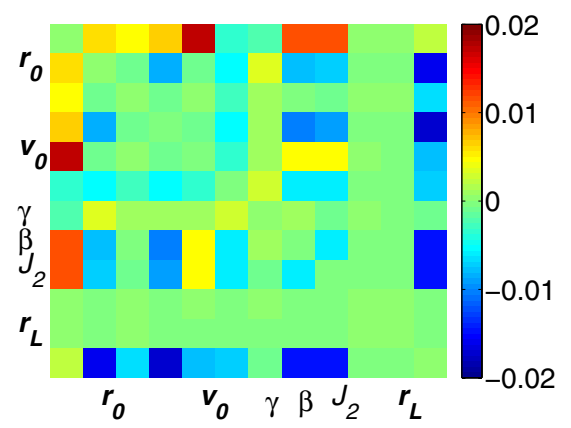

(a)

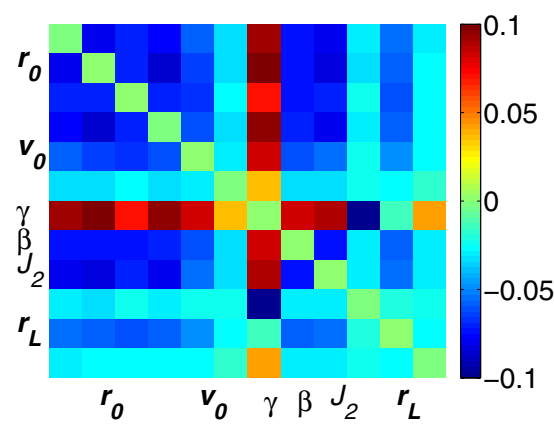

(b)

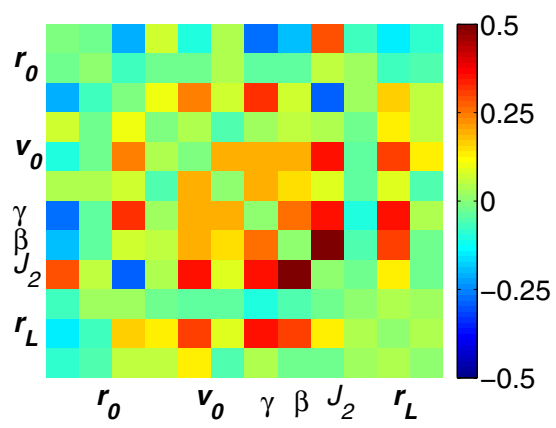

(c)

Fig. 1. Relative difference $\mathbf{\Delta} \mathbf{C}$ between correlation matrices (omitting bias entries) of estimation incorporating initial proper time estimation and bias-only estimation, defined in Eq. (39): a) four-year Mars mission; b) one-month Mercury mission; c) four-year Mercury mission.

the inclusion of the space-time coupling, which can be crucial in the interpretation of the estimation results (Sect. 1).

We compare the estimation where we include the proper time estimation with the estimation where we use the classical approach (i.e. no initial proper time estimation), but add the estimation of range biases. As discussed in Sect. 3.3, the behaviour of the observation partials with respect to the biases is similar to that of the initial proper times. The estimation of range biases is typically done in the analysis of satellite laser ranging (SLR) data analysis, although usually over shorter arcs. In the analyses where we include the estimation of the proper time differences, any existing range bias is absorbed by the proper time difference estimate, so we do not explicitly include the range bias estimation there.

The changes in the entries of the correlation matrix between the time transfer simulations and range simulations are presented relative to one minus the absolute value of the original (rangeonly) correlation matrix $\mathbf{C}_{r}$, so that

$\Delta C_{i j}=\frac{\left|\left(C_{t}\right)_{i j}\right|-\left|\left(C_{r}\right)_{i j}\right|}{1-\left|\left(C_{r}\right)_{i j}\right|}$,

where we implicitly set $\Delta C_{i i}$ to 0 and $\mathbf{C}_{t}$ denotes the correlation matrix of the time transfer simulation that includes the proper time difference estimation. In this comparison criterion, we divide by $1-\left|\left(C_{r}\right)_{i j}\right|$ instead of $\left|\left(C_{r}\right)_{i j}\right|$, since it is not only important to ascertain the absolute changes in the correlation values, but especially to assess the ability of the estimation to decouple two parameters. That is, a change in correlation of 0.01 is crucial if it changes from 0.99 to 1.0 (or vice versa) since it makes the difference between being completely unable and slightly able to decouple the two parameters. Conversely, a change from 0 to 0.01 (or vice versa) is largely irrelevant since the parameters remain almost completely decoupled.

The formal error differences of the estimated parameters with and without the use of the initial proper time difference estimation are shown in Table 1. For the Mars lander, it can clearly be seen that none of the formal errors is significantly affected by the addition of the initial proper time difference estimation, with all relative changes smaller than $1 \%$. The relative change in the correlation matrix is shown in Fig. 1. For the Mars simulation, the difference between the correlation matrices of the two estimations is marginal, as shown in Fig. 1a, with differences around and well below the $1 \%$ level. Although these differences are larger than for the formal error, it still signifies the extremely limited influence of the addition of the relativistic clock effects on the estimation.
Table 1. Change in formal errors (in\%) between estimation from range data to landers with estimated initial proper time difference and estimated bias.

\begin{tabular}{lccc}
\hline \hline & $\begin{array}{c}\text { Mars } \\
(4 \mathrm{y})\end{array}$ & $\begin{array}{c}\text { Mercury } \\
(1 \mathrm{month})\end{array}$ & $\begin{array}{c}\text { Mercury } \\
(4 \mathrm{y})\end{array}$ \\
\hline $\boldsymbol{r}_{0}$ & -0.0138 & -3.51 & 2.56 \\
$\boldsymbol{v}_{0}$ & -0.158 & -2.31 & 6.31 \\
$\gamma$ & 0.0480 & 2.93 & -9.65 \\
$\beta$ & -0.236 & -3.68 & 15.3 \\
$J_{2, \odot}$ & -0.255 & -3.29 & 9.93 \\
$\boldsymbol{r}_{L}$ & 0.0125 & -1.75 & 3.27 \\
\hline
\end{tabular}

The difference in formal error statistics for both the shortand long-duration Mercury lander mission is also given in Table 1. Even for the short Mercury mission, the influence of the space-time dynamics coupling on the formal error statistics is more than an order of magnitude larger than for the Mars lander. For the four-year Mercury mission, the formal error of $\beta$ changes by $15 \%$. The formal estimation errors of $\gamma$ and $J_{2, \odot}$ both change by about $10 \%$. These values show that, even for a longduration mission relatively deep in the gravity well of the Sun, the differences in formal error will be limited. Nevertheless, the observed differences at the $10 \%$ level may warrant the use of the combined initial space-time dynamics estimation, but depend on the degree and manner in which non-Gaussian observation uncertainties and model errors propagate into the estimation error budget.

We show the change in the correlation matrix for the Mercury mission, as quantified by $\mathbf{\Delta} \mathbf{C}$ from Eq. (39), in Figs. $1 b$ and $1 \mathrm{c}$ for the 30-day and 4-year Mercury mission, respectively. As can be seen when comparing these figures to those in Table 1, the maximum values of $\boldsymbol{\Delta} \mathbf{C}$ are substantially larger than the changes in the formal error (as was the case for the Mars mission simulations) by a factor of about three for both the 30-day and 4-year mission. This difference is due to the $1-\left(C_{r}\right)_{i j}$ term in Eq. (39), which causes larger relative changes in $\Delta \mathbf{C}$ for correlations that are closer to 1 (or -1 ). For the four-year mission, relatively large relative changes of up to 0.5 and down to -0.25 are observed. Positive values signify cases where the simulations using the coupled estimation have a larger absolute correlation, see Eq. (39). The largest relative change in correlation occurs between $\beta$ and $J_{2, \odot}$ for the four-year mission, where we observe a change in correlation coefficient from -0.74 to -0.87 . This shows that the actual correlation between the signals of these two parameters is significantly larger than that obtained from 

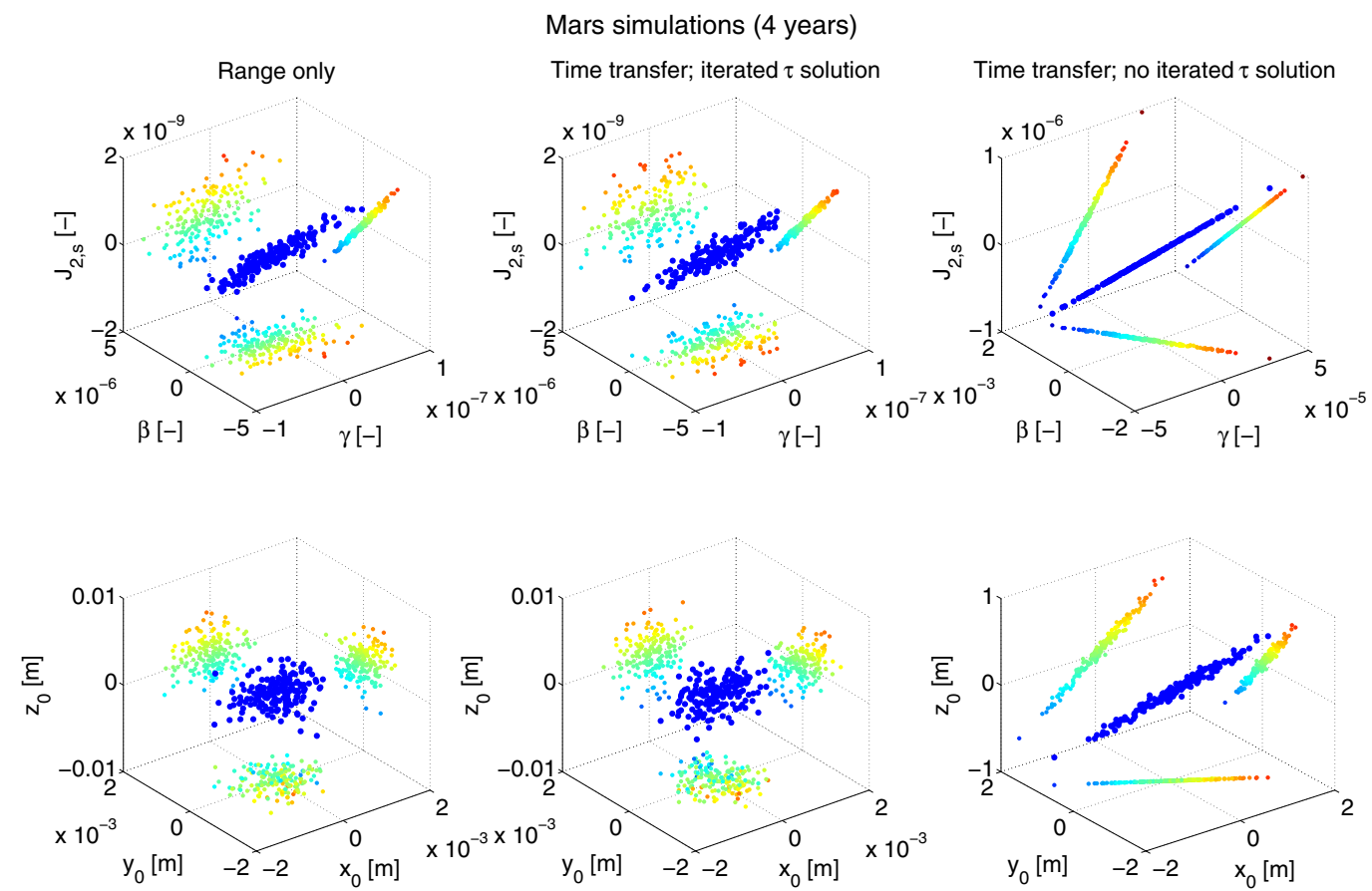

Fig. 2. Estimation errors obtained from numerical simulations for the four-year Mars mission. Panels show estimation error clouds for ideal $t(\tau)$ conversion, iterated $t(\tau)$ conversion, and unupdated a priori $t(\tau)$ conversion. Projections of estimations errors onto the three subplanes are also shown, coloured by error in $J_{2, \odot}(t o p)$ and initial $z$-position (bottom).

the classical approach (without proper time estimation). This indicates that, if the space-time dynamics coupling is omitted in the data analysis process, the correlation between the two parameters that is obtained from the estimation will be substantially more optimistic than the true correlation between the signals from these parameters.

Our results here do not imply that the relativistic clock effects are too weak to be of use for parameter estimation. It instead shows that when using the same data (laser range in these simulations) for orbit determination and time transfer, the resulting orbital accuracy will put stronger constraints on the parameters of interest than the relativistic clock effects. However, this may not be the case for missions where time transfer is only sporadically performed, and orbit determination is also performed using other data types such as Doppler tracking. In these cases, the time transfer observable may be relatively more accurate than the orbital accuracy, potentially increasing the relative strength of the time transfer observables in the estimation. Also, highly accurate clocks carried by orbiting spacecraft can be used for long-arc comparison of ground- and space-based clocks, whereas their orbits are typically estimated over relatively short arcs (hours to days) to prevent the accumulation of (non-conservative) force model errors. As such, the longer duration over which the proper time behaviour could potentially be estimated (depending on the clock's stability), compared to the duration over which the state behaviour can be estimated, may increase the importance of the inclusion of the proper time into the estimation.

\subsection{Influence of a priori and a posteriori calibration}

Having analysed the influence of the space-time dynamics coupling on the estimation's formal errors in Sect. 4.2, we now investigate by direct numerical simulation the influence of omitting this coupling and performing a priori or iterative a posteriori clock calibration.

To this end, we perform an estimation using the simulation settings defined in Sect. 4.1. We perturb the parameters at their current level of uncertainty and use an iterative least-squares method to estimate these parameters. We perform three sets of simulations for both the Mars and Mercury missions:

1. Estimation in which the true $t(\tau)$ conversion for each observer is known perfectly.

2. Estimation in which a $t(\tau)$ conversion is recomputed following each iteration of the estimation (a posteriori clock calibration).

3. Estimation in which a $t(\tau)$ conversion based upon the a priori perturbed parameter estimate is used.

From these simulations, we are able to determine the parameter estimation error that is made due to mismodelled relativistic clock effects when using the typical orbit determination method.

We find no difference in our results for the one-month Mercury lander mission between the three cases because the estimation errors of even the ideal case are larger than the initial perturbation in the parameter set. In other words, for the short Mercury mission, the tracking data inversion does not result in an improved parameter estimation compared to the a priori uncertainty.

For both the Mars and Mercury four-year tracking simulations, the resulting uncertainties for 100 simulations are shown in Figs. 2 and 3, respectively. The error clouds of both the initial position of Mars/Mercury and the parameters $\gamma, \beta$, and $J_{2, \odot}$ are shown. In these figures, the projections of these clouds onto the three planes are also shown. We note that our results for $\gamma$ for the four-year Mars simulation are similar to the estimation results obtained by Turyshev et al. (2010; error of $1.4 \times 10^{-7}$ after 3 years; $7.9 \times 10^{-8}$ after 6 years), who perform simulations of 

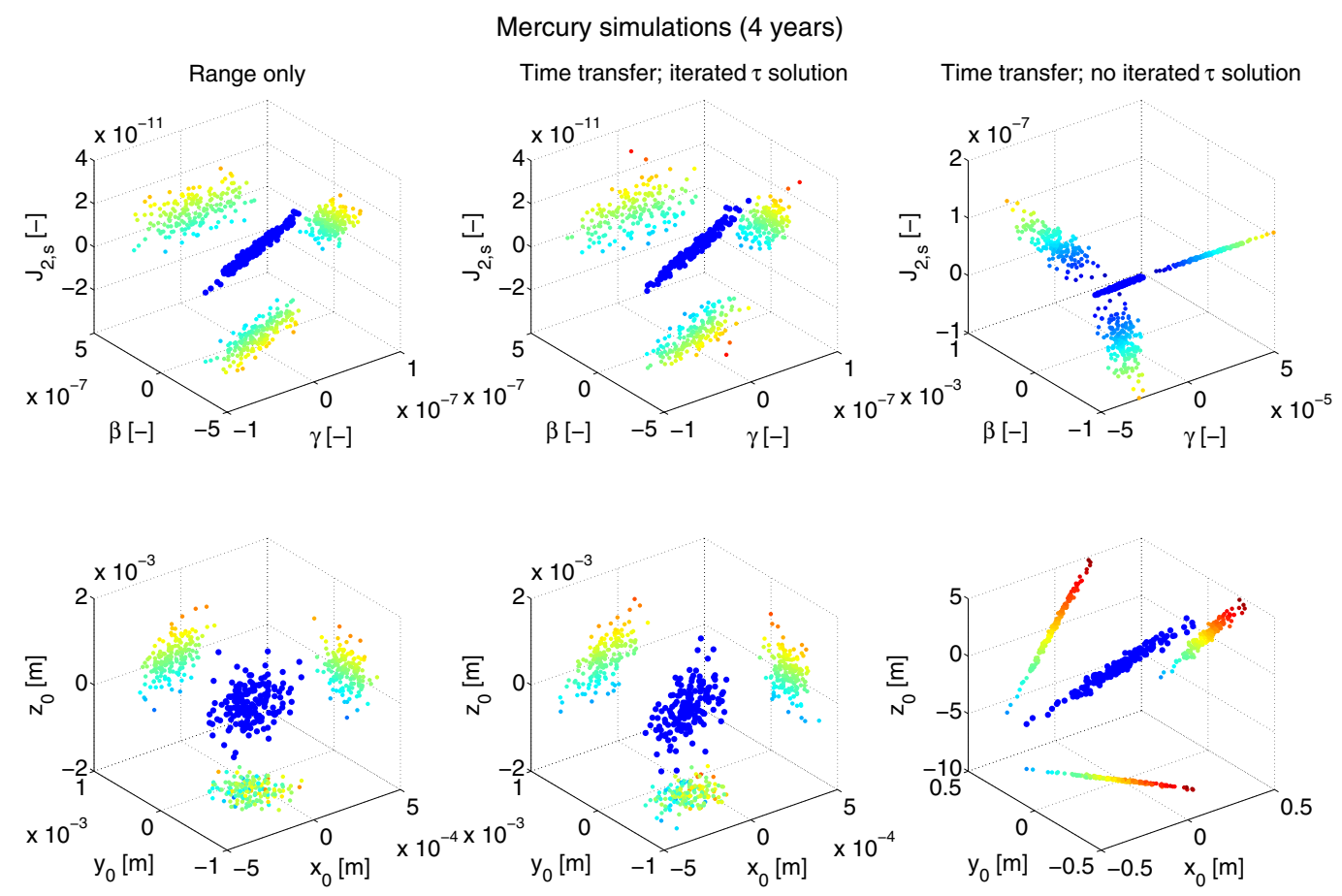

Fig. 3. Estimation errors obtained from numerical simulations for the four-year Mercury mission. Panels show estimation error clouds for ideal $t(\tau)$ conversion, iterated $t(\tau)$ conversion, and unupdated a priori $t(\tau)$ conversion. Projections of estimations errors onto the three subplanes are also shown, coloured by error in $J_{2, \odot}($ top $)$ and initial $z$-position (bottom).

laser ranging to Phobos. Our results for $\beta$ and $J_{2}$ are about an order of magnitude more optimistic, most likely due to our smaller set of estimated parameters and resultantly reduced correlations. However, we reiterate that our goal here is not so much to provide highly accurate absolute estimates for attainable estimation accuracy, but to compare different data analysis techniques.

We observe differences of orders of magnitude between cases 2 and 3. That is, the errors in the a priori $t(\tau)$ conversion are sufficiently large to drive the estimation to erroneous results. This influence was not observed in the previous section where the truth and estimation model were assumed to be equal. This indicates that the use of a priori time ephemerides, for instance those of (Irwin \& Fukushima 1999; Fienga et al. 2009), for the data analysis of planetary laser ranging missions can result in substantial degradations of the estimation quality. In general, this will be true if the a priori orbital and parameter uncertainty is much greater than the range measurement uncertainty.

It must be noted, however, that the simulations shown here are performed using purely Gaussian noise on the observations. As a result, the estimation accuracy shown here for cases 1 and 2 is substantially better than what will be obtainable from real tracking data, which will include time-correlated and nonGaussian noise. Differences between true and formal errors for interplanetary (radiometric) tracking data inversion are typically around a factor 5-10 (e.g. Marty et al. 2009; Konopliv et al. 2011).

The difference between cases 1 and 2 is insignificant for the four-year Mars mission. This is consistent with the fact that we found formal error differences at the $0.1 \%$ level in Sect. 4.2. For the four-year Mercury mission, we find small but statistically significant differences between the error distributions of cases 1 and 2 with around $10 \%$ degradation in estimation error for case 2 compared to case 1. Again, this is consistent with the results presented in the previous section, which showed differences in formal errors of around 5-10\% for simulations with and without the inclusion of the coupling.

\section{Conclusions}

We have derived an estimation framework with which the influence of relativistic clock effects on interplanetary range and time transfer data analysis can be included on an equal footing with relativistic influence on translational dynamics. The main theoretical results of our development are Eqs. (22) and (36), which are extended versions of the classical equations used in orbit determination. Our approach allows the degree to which relativistic clock effects can be used to improve parameter estimation and orbit determination to be directly quantified. Also, it provides a reliable quantification of the correlation between the influence of relativistic clock effects and translational dynamics on the time transfer observable, allowing for robust tracking data analysis for a broad range of mission profiles.

We have simulated one-way data to Mars and Mercury landers and used the simulated data for a covariance analysis, assessing the possibilities of using these data for the determination of $\gamma, \beta$, and $J_{2} \odot$. We analyse the use of both range measurements (including the estimation of range biases) and time transfer (including the estimation of an initial proper time difference). The inclusion of the estimation of the proper time difference between Earth stations and a Mars lander has a negligible influence on both the correlations between the estimated parameters and their formal errors, with differences in the formal errors of around $0.1 \%$ observed. For a Mercury lander this formal error difference rises to $1 \%$ for a one-month mission and $10 \%$ for a four-year mission. However, we find a significantly greater relevant degree of change in the correlation matrix (as quantified by $\Delta \mathbf{C})$ than for the formal errors, which can be of importance for the interpretation of the robustness of the results, as well as any statistical analysis of postfit residuals. 
These results indicate that the influence of our estimated parameter set on the translational dynamics is much stronger than on the proper time rate owing to the $c^{-2}$ term in Eq. (8). For spacecraft state estimation, where the translational state is typically determined over short arcs, a highly stable clock may allow substantially modified parameter estimation quality.

We have shown by direct numerical simulation that using an a priori time ephemeris with sufficiently low accuracy can result in orders of magnitude degradation of the true error distribution compared to the formal errors. Recomputing the proper-tocoordinate time conversion for each observer after each iteration of the least-squares estimation results in a degradation similar to the changes that are observed in the formal errors when including the proper time estimation.

Acknowledgements. Dominic Dirkx is financed by the FP7 ESPaCE project, financially supported by the EC FP7 Grant Agreement 263466. The authors thank Alexander Polnarev of the Queen Mary University of London, Sergei Kopeikin of the University of Missouri, and anonymous reviewers for their insightful input that significantly improved the clarity and quality of the manuscript.

\section{References}

Angélil, R., Saha, P., Bondarescu, R., et al. 2014, Phys. Rev. D, 89, 064067 Birnbaum, K. M., Chen, Y., \& Hemmati, H. 2010, in SPIE Conf. Ser., 7587 Bjerhammar, A. 1985, Bull. Geod., 59, 207

Blanchet, L., Salomon, C., Teyssandier, P., \& Wolf, P. 2001, A\&A, 370, 320

Bloom, B. J., Nicholson, T. L., Williams, J. R., et al. 2014, Nature, 506, 71

Brumberg, V. A. 1972, Relativistic Celestial Mechanics (in Russian) (Nauka), 384

Brumberg, V. A. 1991, Essential relativistic celestial mechanics (CRC Press) (Bristol, England and New York: Adam Hilger)

Degnan, J. 2002, J. Geodyn., 34, 551

Dehant, V., Le Maistre, S., Rivoldini, A., et al. 2011, Planet. Space Sci., 59, 1069

Delva, P., Meynadier, F., Le Poncin-Lafitte, C., Laurent, P., \& Wolf, P. 2012, in European Frequency and Time Forum (EFTF), 2012, 28

Delva, P., Hees, A., Bertone, S., Richard, E., \& Wolf, P. 2015, CQG, 32, 232003

Deng, X.-M., \& Xie, Y. 2013a, RA\&A, 13, 1225

Deng, X.-M., \& Xie, Y. 2013b, MNRAS, 431, 3236

Deng, X.-M., \& Xie, Y. 2015, New Astron., 35, 36

Dirkx, D. 2015, Ph.D. Thesis, Delft University of Technology, The Netherlands

Dirkx, D., Vermeersen, L., Noomen, R., \& Visser, P. 2014, Planet. Space Sci., 99,84
Dirkx, D., Noomen, R., Visser, P., Bauer, S., \& Vermeersen, L. 2015, Planet. Space Sci., 117, 159

Duchayne, L., Mercier, F., \& Wolf, P. 2009, A\&A, 504, 653

Ely, T. A., Seubert, J., \& Bell, J. 2014, in SpaceOps Conference, AIAA 2014-1856

Exertier, P., Samain, E., Bonnefond, P., \& Guillemot, P. 2010, Adv. Space Res., 46, 1559

Fienga, A., Laskar, J., Morley, T., et al. 2009, A\&A, 507, 1675

Fienga, A., Laskar, J., Exertier, P., Manche, H., \& Gastineau, M. 2015, Celest. Mech. Dyn. Astron., 123, 325

Folkner, W. M., Williams, J. G., Boggs, D. H., Park, R. S., \& Kuchynka, P. 2014, Interplanetary Network Progress Report, 196, C1

Hees, A., Lamine, B., Reynaud, S., et al. 2012, Class. Quant. Grav., 29, 235027

Iorio, L. 2012, J. Cosmol. Astroparticle Phys., 7, 1

Irwin, A. W., \& Fukushima, T. 1999, A\&A, 348, 642

Klioner, S. A. 1992, Celest. Mech. Dyn. Astron., 53, 81

Klioner, S. A. 2008, A\&A, 478, 951

Konopliv, A. S., Asmar, S. W., Folkner, W. M., et al. 2011, Icarus, 211, 401

Kopeikin, S., \& Vlasov, I. 2004, Phys. Rep., 400, 209

Kopeikin, S., Efroimsky, M., \& Kaplan, G. 2011, Relativistic Celestial Mechanics of the Solar System (Wiley VCH)

Kopeikin, S. M., \& Schäfer, G. 1999, Phys. Rev. D, 60, 124002

Linet, B., \& Teyssandier, P. 2002, Phys. Rev. D, 66, 024045

Marty, J. C., Balmino, G., Duron, J., et al. 2009, Planet. Space Sci., 57, 350

Mayrhofer, R., \& Pail, R. 2012, in Geodesy for Planet Earth (Springer), 231

Mecheri, R., Abdelatif, T., Irbah, A., Provost, J., \& Berthomieu, G. 2004, Sol Phys., 222, 191

Minazzoli, O., \& Chauvineau, B. 2011, Class. Quant. Grav., 28, 085010

Misner, C., Thorne, K., \& Wheeler, J. 1973, Gravitation (W. H. Freeman)

Montenbruck, O., \& Gill, E. 2000, Satellite Orbits: Models, Methods, and Applications, Physics and Astronomy Online Library (Springer Verlag)

Moyer, T. D. 2005, Formulation for observed and computed values of Deep Space Network data types for navigation (John Wiley \& Sons), 3

Müller, J., Soffel, M., \& Klioner, S. A. 2008, J. Geodesy, 82, 133

Nelson, R. A. 2011, Metrologia, 48, 171

Pan, J.-Y., \& Xie, Y. 2015, RA\&A, 15, 281

Pearlman, M. R., Degnan, J. J., \& Bosworth, J. M. 2002, Adv. Space Res., 30, 135

Petit, G., \& Wolf, P. 1994, A\&A, 286, 971

Prestage, J., \& Weaver, G. 2007, Proceedings of the IEEE, 95, 2235

Schärer, A., Angélil, R., Bondarescu, R., Jetzer, P., \& Lundgren, A. 2014, Phys. Rev. D, 90, 123005

Soffel, M. H. 1989, Relativity in Astrometry, Celestial Mechanics and Geodesy (Springer-Verlag)

Soffel, M., Klioner, S. A., Petit, G., et al. 2003, AJ, 126, 2687

Turyshev, S. G., Farr, W., Folkner, W. M., et al. 2010, Exp. Astron., 28, 209

Verma, A. K., Fienga, A., Laskar, J., Manche, H., \& Gastineau, M. 2014, A\&A, 561, A115 